\title{
CDC Alert for Hospital Infections due to Candida Auris Multiresistant
}

\author{
Dra Mirta D Ambra* \\ University of Buenos Aires, Argentina \\ *Corresponding author: Dra Mirta D Ambra, Executive Board WAMS Epidemiology, University of Buenos Aires, Argentina. \\ To Cite This Article: Dra Mirta D Ambra. CDC Alert for Hospital Infections due to Candida Auris Multiresistant. Am J Biomed Sci \& Res. 2019 - \\ 3(1). AJBSR.MS.ID.000625. DOI: 10.34297/AJBSR.2019.03.000625
}

Received: April 21, 2019 | Published: May 14, 2019

\begin{abstract}
Summary
The Centers for Disease Control and Prevention (CDC) has received reports from international health centers that Candida auris multidrug resistant yeast is causing health-associated invasive infections with high mortality. Some strains of $C$. auris have high minimum inhibitory concentrations (MIC) to the three main classes of antifungals, severely limiting treatment options. C. auris requires specialized methods for its identification and could be mistakenly identified as another yeast when the procedure is based on traditional biochemical methods. The CDC is aware that a strain of $C$. auris was detected in the United States in 2013 as part of ongoing surveillance. The experience outside the United States suggests that $C$. auris has a high potential to cause outbreaks in health centers. Given the presence of $C$. auris in nine countries on four continents since 2009, the CDC is alerting health centers in the United States to be on the lookout for C. auris infections in patients (Figure 1).
\end{abstract}

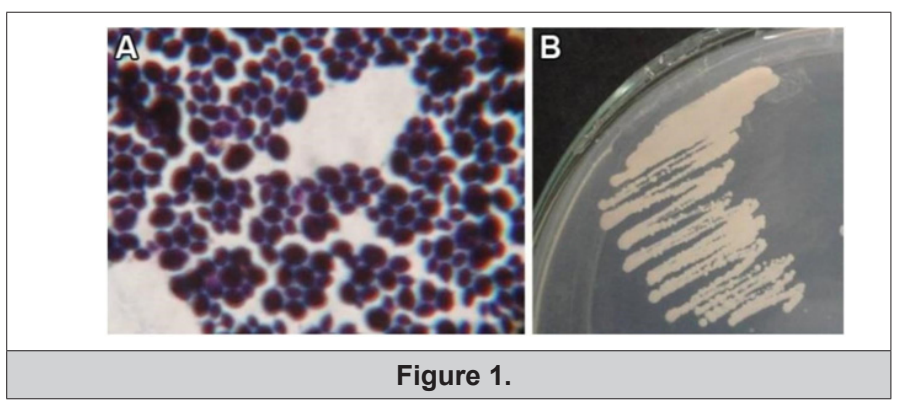

\section{Background}

Candida auris is a multi-drug emergent yeast (MDR) that can cause invasive infections and is associated with high mortality. It was first described in 2009 after being isolated in the secretion of the external ear of a patient in Japan 1. Since the 2009 report, $C$. auris infections, specifically fungemia, have been reported in South Korea 2, 3 India, South Africa 4, and Kuwait 5. Although published reports are not available, $C$. auris. It has also been identified in Colombia, Venezuela, Pakistan and the United Kingdom.

It is unknown why Candida auris has recently emerged in so many different places. The molecular typing of the strains carried out by the CDC suggests that the isolated elements are highly related within a country or region, but in different continents 6 . The earliest known infection with Candida auris, based on retrospective tests, occurred in South Korea in 1996. Candida auris may not represent a new organism as well as one that has recently appeared in various clinical settings. Although the causes of such an emergency are unknown, they may include new selective or increasing pressures using antifungals in humans, animals or the environment.

Candida auris infections have been more commonly acquired in the hospital and occurred after several weeks of hospital stay in a patient. It has been reported that Candida auris causes bloodstream infections, wound infections, and otitis 2 . It has also been cultured from the urine and respiratory tract; however, it is unknown whether the isolation of these sites has represented infection or colonization in each case. It has been documented that Candida auris can cause infections in patients of all ages. It was found that patients have risk factors like those described for infections with other varieties of Candida spp. 6, 7, including: diabetes mellitus, recent surgery, recent antibiotics, and the presence of central venous catheters. 3. Co-infection with another variety of Candida spp. Has also been reported. and of Candida auris while the patient was being treated with antifungals. Two Although there are no 
cut-off points for the minimum inhibitory concentration (MIC) established for Candida auris at this time, the resistance test of an international collection of isolated microorganisms performed by CDC showed that almost all are highly resistant to fluconazole on the basis of the cut-off points established for other varieties of Candida spp. More than half of the Candida auris isolates were resistant to voriconazole, one third were resistant to amphotericin $\mathrm{B}(\mathrm{MIC} \geq 2)$, and a few were resistant to echinocandins. Some strains have demonstrated high minimum inhibitory concentrations for the three main azole antifungal classes, including, echinocandins, and polyenes, indicating that treatment options would be limited.

Candida auris is phenotypically like Candida haemulonii 1. Commercially available biochemical-based tests, including API and VITEK-2 strips, which are used in many US laboratories to identify fungi, cannot differentiate Candida auris from other related species. Due to these challenges, clinical laboratories have mistakenly identified the organism as Candida haemulonii and Saccharomyces cerevisiae. Some clinical laboratories do not fully identify all candida species, and isolation of Candida auris has been reported as "another Candida spp." Clinicians, the State and public health laboratories must be aware of the presence of this organism and of the limitations of its identification. At least two countries have already described outbreaks of health infection by Candida auris and colonization involving more than 30 patients each. The analysis of the analysis of the isolates of these groups demonstrates a high degree of clonality within the same hospital, this supports the idea that organisms are transmitted within these health centers. The precise mode of transmission within health care facilities is not known. However, the experience during these outbreaks suggests that Candida auris could contaminate the environment of the rooms of colonized or infected patients. Good infection control practices and cleanliness of the environment can help prevent transmission.

\section{Interim Recommendations}

The CDC is concerned that Candida auris will appear in new places, including the United States. The CDC and its partners continue to work closely, and new information will be provided as soon as it becomes available.

\section{CDC recommends the following actions for installation and United States health laboratories}

Health centers that suspect that they have a patient with Candida auris infection should contact the state/local public health authorities and the CDC (candidaauris@cdc.gov) - Reporting.

\section{Laboratory diagnosis}

Diagnostic devices based on the desorption/ionization laserassisted matrix or MALDI-TOF can differentiate Candida auris, but not all devices currently include the Candida auris in their reference database to allow detection. Molecular methods based on the sequencing of the D1-D2 region of 28S rDNA can also identify Candida auris. The CDC made requests to laboratories that identify
Candia auris in the United States to notify their state or local health departments and the CDC (candidaauris@cdc.gov).

\section{Infection Control}

Until more information becomes available, health centers should place patients with colonization or Candida auris infection in individual rooms and health personnel should use standard precautions for contact precautions. In addition, state or local health authorities and CDC should be consulted about the need for additional interventions to prevent transmission. The CDC is working with national and international partners to develop a definitive infection control guide.

\section{Cleanliness of the environment}

Anecdotal reports suggest that Candida auris can persist in the environment. Health centers that have patients with infection or colonization by Candida auris should guarantee the daily cleaning in depth and the disinfection of the rooms of these patients using a disinfectant for hospital use registered by the EPA with a warning for fungi.

\section{Conclusion}

Antifungal resistance of $C$. auris: There are still no cut-off points for the susceptibility of $C$. auris to antifungals. This species is highly resistant to fluconazole (MIC $90>64 \mathrm{mg} / \mathrm{L}$ ) and one third of the isolates have an elevated MIC to voriconazole ( $\geq 2 \mathrm{mg} / \mathrm{L}$ ) and amphotericin B. Few strains have high MICs to echinocandins. Its profile of resistance to the three large families of antifungals limits the therapeutic alternatives [1]. This species is genetically related to $C$. haemulonii, which has intrinsic resistance to amphotericin $\mathrm{B}$ and fluconazole. So far, the mechanisms of resistance are not clearly known.

Apparently, it would be inducible by selection pressure producing rapid mutational changes. A recent genetic study showed that this species would have unique copies of several genes related to resistance to antifungals such as ERG3, ERG11, FKS1, FKS2 and FKS3 as well as a higher proportion of genes from families of transporters ABC and MSF (efflux pumps), that could explain multi resistance. It is probable that the unmediated use of antifungals has resulted in the emergence of $C$. auris as a successful multi-resistant pathogen. Given the high MICs to amphotericin B, echinocandins should be used as first line therapy, with antifungal susceptibility test. In addition, antifungal susceptibility should be monitored in patients infected or colonized by this agent. The potentially devastating impact of invasive infections by this multiresistant species in both patients and clinical services should not be underestimated. It is necessary to continue contributing information to the knowledge of the global epidemiology of this emerging infection and how to prevent it.

\section{References}

1. Shawn Lockhart, An expert on fungal infections at the Centers for Disease Control and Prevention with a microscope slide of "Candida auris". 